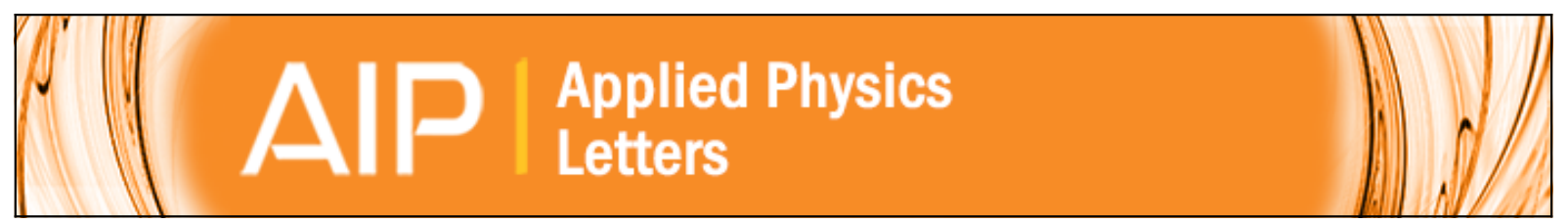

Influence of crystal mosaicity on axial channeling effects and lattice site determination of impurities

B. De Vries, U. Wahl, S. Ruffenach, O. Briot, and A. Vantomme

Citation: Applied Physics Letters 103, 172108 (2013); doi: 10.1063/1.4826705

View online: http://dx.doi.org/10.1063/1.4826705

View Table of Contents: http://scitation.aip.org/content/aip/journal/apl/103/17?ver=pdfcov

Published by the AIP Publishing

$\underset{\substack{A \text { coubshinge } \\ \text { R }}}{R}$ Re-register for Table of Content Alerts 


\title{
Influence of crystal mosaicity on axial channeling effects and lattice site determination of impurities
}

\author{
B. De Vries, ${ }^{1}$ U. Wahl, ${ }^{2}$ S. Ruffenach, ${ }^{3}$ O. Briot ${ }^{3}$ and A. Vantomme ${ }^{1, a)}$ \\ ${ }_{1}^{1}$ Instituut voor Kern- en Stralingsfysica, KU Leuven, 3001 Leuven, Belgium \\ ${ }^{2}$ Campus Tecnológico e Nuclear, Instituto Superior Técnico, Universidade de Lisboa, \\ 2686-953 Sacavém, Portugal \\ ${ }^{3}$ GES, Université de Montpellier II, 34095 Montpellier, France
}

(Received 3 August 2013; accepted 11 October 2013; published online 23 October 2013)

\begin{abstract}
Using the electron emission channeling and Rutherford backscattering/channeling techniques, the influence of the mosaicity of a thin film on the axial channeling of charged particles was investigated. It is found that crystal mosaicity leads to a broadening and a degradation of the experimental channeling effects. This phenomenon, which is shown to be of major importance when assessing the lattice site of impurities in a single crystal, can be modeled quantitatively by using the mosaic tilt and twist components derived from X-ray rocking curve scans. As a case study, we illustrate that our approach allows to accurately determine the lattice site of Er in AlN, despite the significant influence of mosaicity on the channeling measurements. (C) 2013 AIP Publishing LLC. [http://dx.doi.org/10.1063/1.4826705]
\end{abstract}

The channeling of charged particles is widely used to investigate single-crystalline materials. In combination with ion beam techniques such as Rutherford backscattering spectrometry (RBS), particle-induced X-ray emission (PIXE), or nuclear reaction analysis (NRA), ion channeling is a powerful tool to examine the crystal quality of such materials or to obtain direct information on the lattice sites occupied by impurities. One particularly sensitive lattice location technique is emission channeling (EC), which is based on the channeling of charged particles emitted in the decay of radioactive impurities. ${ }^{1}$

Most theoretical treatments of channeling ignore crystal imperfections or only treat point defects, e.g., by the addition of a scattering contribution to the theoretical model for a perfect crystal, which accounts for the collisions of channeled particles with point defects inside the channels. ${ }^{1,2}$ The influence of extended defects is, however, far less studied. One specific example of such long-range crystal disorder is crystal mosaicity, e.g., in a (hetero-)epitaxial thin film which consists of single-crystalline domains that are separated by narrow regions with high dislocation densities. Consequently, the domains are slightly misoriented with respect to each other and to the substrate. The misorientation of a crystallite can be described by a tilt (out-of-plane rotation) and twist (in-plane rotation) component (Fig. 1). So far, ion channeling studies of mosaic layers-both theoretical and experimental-have been limited to the investigation of the channeling effect along the surface normal direction. ${ }^{3-9}$ However, as will be shown below, the surface normal channeling effect is only influenced by the mosaic tilt component. In this work, we will extend the existing model to non-perpendicular channeling directions, which will also probe the twist component. This model will subsequently be used to explain anomalous EC and Rutherford backscattering/channeling (RBS/C) results in thin AlN layers.

\footnotetext{
a)Andre.Vantomme@fys.kuleuven.be
}

For a certain channeling axis, the microscopic spread in orientation gives rise to a macroscopic distribution function for that axis, as schematically shown in Fig. 1 for a crystallographic direction that is inclined by an angle $\theta$ with respect to the surface normal, with the two-dimensional distribution function shown in grey. We define the $x$-axis to be perpendicular to the channeling axis and pointing towards the surface normal, while the $y$-axis is perpendicular to both the channeling axis and the $x$-axis. The width of the projection on the $x$-axis will only depend on the spread in mosaic tilt $W_{\text {tilt, }}$ irrespective of the inclination angle $\theta$. On the other hand, the width of the $y$-axis projection will be determined by a superposition of tilt $W_{\text {tilt }}$ and twist $W_{\text {twist, }}$ which varies as a function of $\theta$. An empirical model that describes this superposition has been developed by Srikant et al. ${ }^{10}$ This spread in orientation will evidently have repercussions on channeling experiments: theoretically, if the mosaic domains are large enough so that most particles will channel within one single-crystalline domain, the resulting channeling effect simply consists of the convolution of the channeling effect

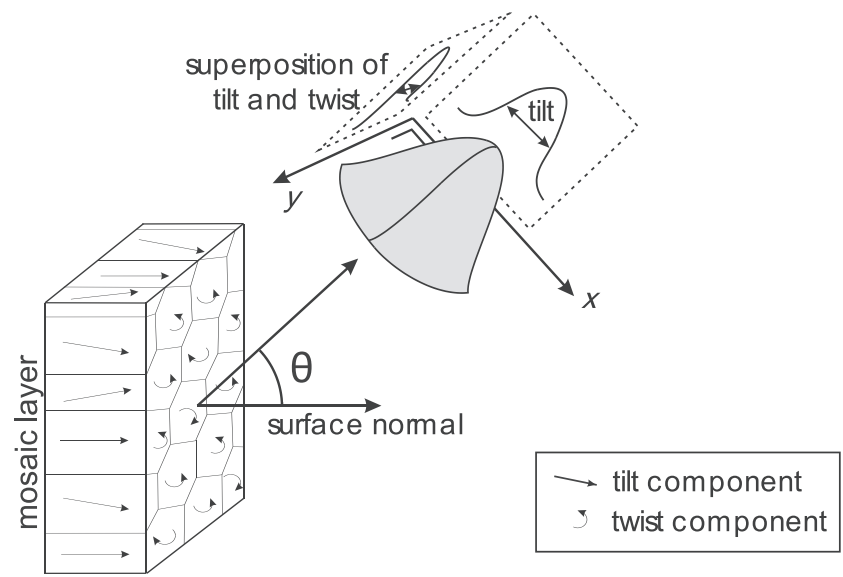

FIG. 1. Schematic of the macroscopic distribution of a crystallographic axis due to the microscopic mosaic spread. 
for a perfect crystal with the distribution function of the channeling axis. This will result in a broadening of the experimental channeling patterns and a degradation of the channeling yields compared to those for a perfect crystal. However, this effect will only be visible experimentally if the mosaic spread is of the same order of magnitude or larger than the experimental angular resolution. If so, it is essential to take the mosaicity into account during the analysis of the experimental data in order to correctly interpret the results, both in a qualitative (identification of the correct lattice site) and quantitative (correct fractions) way. Conversely, when the mosaic spread becomes significantly larger than the full-width at half maximum (FWHM) of the channeling effects-in the ultimate case, a polycrystalline sample-the measurement merely reflects the crystalline structure, without providing reliable information on the lattice site.

The sample consists of an AIN layer grown by metalorganic chemical vapor deposition (MOCVD) onto a sapphire substrate, with a buffer layer of AlGaN in between. The layer composition and thickness were analyzed by means of RBS (not shown) using a ${ }^{4} \mathrm{He}^{+}$beam with random incidence. In order to resolve the energy signals of ${ }^{4} \mathrm{He}$ particles backscattered from $\mathrm{Al}$ and $\mathrm{Ga}$, a beam energy of $3.5 \mathrm{MeV}$ and a detection angle of $168^{\circ}$ were used. In addition, complete angular scans were performed for the [0001], [2113], and [1101] directions, giving additional information on the crystalline quality.

In order to assess the tilt distribution in the AlN film, we measured the high-resolution X-ray rocking curves ( $\omega$-scan) of the (0002) and (0004) reflections using a $\mathrm{Cu}$ source and a monochromator with four $\mathrm{Ge}(002)$ crystals such that only the $\mathrm{K}_{\alpha 1}$ radiation is selected. The reflected $\mathrm{X}$-rays pass through $1 \mathrm{~mm}$ and $0.6 \mathrm{~mm}$ slits before being detected by a scintillation detector. The instrumental resolution was $0.005^{\circ}$ FWHM. In order to determine the twist, a series of skew-symmetric rocking curve reflections was performed.

Electron emission channeling is based on the fact that electrons emitted during the decay of radioactive isotopes are guided by the potential of rows and planes of atoms while traveling through a crystalline solid. ${ }^{1}$ By measuring the angle-dependent emission yields with a position-sensitive detector and fitting them to theoretically calculated patterns, a precise determination of the lattice site(s) occupied by the emitting isotopes is possible. ${ }^{11}$ In this work we have used the radioactive probe isotope ${ }^{167} \mathrm{Tm}\left(t_{1 / 2}=9.25 \mathrm{~d}\right)$, which was introduced in the AlN sample by $30 \mathrm{keV}$ ion implantation at the ISOLDE facility of CERN to a fluence of $1.9 \times 10^{13} \mathrm{~cm}^{-2}$. The ${ }^{167} \mathrm{Tm}$ isotope decays into the isomeric excited state ${ }^{167 \mathrm{~m}} \operatorname{Er}\left(t_{1 / 2}=2.27 \mathrm{~s}\right)$. In the subsequent decay to the ground state, conversion electrons are emitted, which were used as channeling particles. The electron channeling patterns were measured in the as-implanted state, after $10 \mathrm{~min}$ isochronal annealing steps at $600^{\circ} \mathrm{C}$ and $900^{\circ} \mathrm{C}$, and after tube furnace annealing for $10 \mathrm{~min}$ at $1050^{\circ} \mathrm{C}$ in $\mathrm{N}_{2}$ under atmospheric pressure. More information on emission channeling experiments with the probe ${ }^{167 \mathrm{~m}} \mathrm{Er}$ in $\mathrm{GaN}$ and $\mathrm{Si}$ can be found in Refs. 12-14.

From the random RBS spectrum, a thickness for the top AlN layer of $3500 \AA$ was deduced, while the buffer layer consists of $\mathrm{Al}_{0.2} \mathrm{Ga}_{0.8} \mathrm{~N}$ with a thickness of $5500 \AA$. Table I
TABLE I. RBS/C half-angles and minimum yields of angular scans of 3.5 MeV ${ }^{4} \mathrm{He}$ particles backscattered from $\mathrm{Al}$ in the AlN film: experimentally determined half angles $\psi_{1 / 2}$, theoretically calculated Barrett half angles $\psi_{1 / 2}^{\mathrm{B}}$, corrected half angles $\psi_{1 / 2}^{\text {mos }}$ including the broadening due to crystal mosaicity, experimental minimum yields $\chi_{\text {min }}$, and theoretically calculated Barrett minimum yields $\chi_{\min }^{\mathrm{B}}$.

\begin{tabular}{lcccccc}
\hline \hline axis & $\theta$ & $\psi_{1 / 2}$ & $\psi_{1 / 2}^{\mathrm{B}}$ & $\psi_{1 / 2}^{\operatorname{mos}}$ & $\chi_{\min }(\%)$ & $\chi_{\min }^{\mathrm{B}}(\%)$ \\
\hline$[0001]$ & $0^{\circ}$ & $0.48^{\circ} \pm 0.05^{\circ}$ & $0.42^{\circ}$ & $0.44^{\circ}$ & $5.5 \pm 0.5$ & 1.6 \\
{$[\overline{2} 113]$} & $31.6^{\circ}$ & $0.54^{\circ} \pm 0.15^{\circ}$ & $0.31^{\circ}$ & $0.51^{\circ}$ & $36 \pm 2$ & 3.4 \\
{$[\overline{1} 101]$} & $46.8^{\circ}$ & $0.80^{\circ} \pm 0.30^{\circ}$ & $0.27^{\circ}$ & $0.61^{\circ}$ & $68 \pm 2$ & 4.3 \\
\hline \hline
\end{tabular}

lists the experimentally determined half-angles $\psi_{1 / 2}$ and minimum yields $\chi_{\min }$ for channeling along three different crystal axes in AlN. The theoretically expected half-angles angles $\psi_{1 / 2}^{\mathrm{B}}$ and minimum yields $\chi_{\min }^{\mathrm{B}}$ calculated according to the Barrett formalism ${ }^{15}$ are also shown. While the half-angle and minimum yield of the [0001] surface direction are quite close to the theoretically expected values, the deviation between theory and experiment becomes progressively larger with increasing angle $\theta$. Especially the fact that the experimental half-angles increase with increasing inclination angle, while the theoretical half-angles decrease, and that the experimental minimum yields are considerably larger than the theoretical ones, are already a strong indication that the AlN layer does not possess a perfect single-crystalline structure but is subject to mosaic spread.

From the rocking curve ( $\omega$-scan) of the AIN (0002) reflection, a FWHM of $0.25^{\circ} \pm 0.01^{\circ}$ was found (not shown). This width is the result of a combination of the spread in mosaic tilt, the lateral coherence of the X-rays, and the instrumental resolution. Since the latter is of the order of $0.005^{\circ}$, it can be neglected. Considering that the incoherence broadening decreases with increasing reflection order, its magnitude can be determined by comparing the widths of the (0002) and (0004) rocking curve scans. Since a FWHM of $0.24^{\circ} \pm 0.01^{\circ}$ was measured for the (0004) reflection, the incoherent broadening must be smaller than $0.02^{\circ}$. Therefore, it can also be neglected, and the broadening observed in the rocking curve scan can be attributed solely to mosaic tilt.

The in-plane twist component, on the other hand, is less straightforward to determine. In principle, it can be measured directly by a rocking curve scan, but only in the geometry with grazing incident and exit beams. ${ }^{16}$ A more desirable approach is to determine the twist by the extrapolation of the FWHM of a series of skew-symmetric rocking curve reflections, ${ }^{10}$ since this allows a direct measurement of the superposition of tilt and twist as a function of inclination angle $\theta$. It is exactly this superposition that will be needed to determine the broadening of channeling effects in the $y$ direction (see Fig. 1). Figure 2 shows the FWHM of six skewsymmetric rocking curves as a function of inclination angle. Fitting the data points with the model of Srikant et al. ${ }^{10}$ (solid line) shows that the twist component $\left(1.48 \pm 0.11^{\circ}\right)$ is much larger than the tilt, which qualitatively agrees with previous studies. ${ }^{10,17}$ It is also considerably larger than the typical resolution of an EC experiment, which is $\sim 0.24^{\circ}$. Therefore, the influence of the mosaicity in this sample should be detectable by the EC technique. 


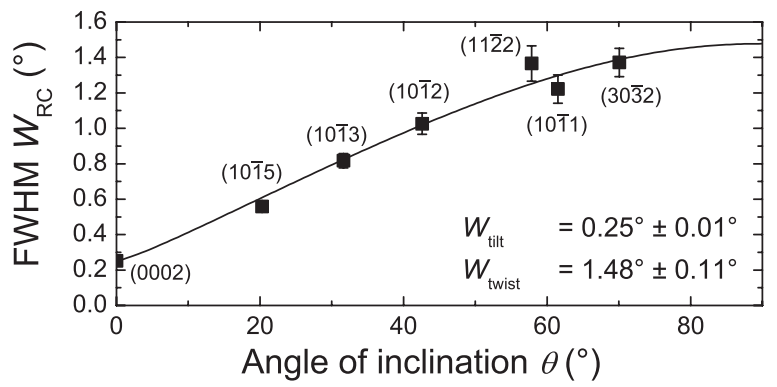

FIG. 2. FWHM $W_{\mathrm{RC}}$ of ( $h \mathrm{kil}$ ) rocking curves as a function of inclination angle with respect to the surface normal. The solid line is a fit with the model of Srikant et $_{\text {al. }}{ }^{10}$

Figures 3(a)-3(d) show the normalized angular emission yields of ${ }^{167 \mathrm{~m}} \mathrm{Er}$ conversion electrons around the [0001], [1102], [2113], and [11101] axes after the $900^{\circ} \mathrm{C}$ annealing step. The inclination angles of these axes with respect to the surface normal are $0.0^{\circ}, 28.0^{\circ}, 31.6^{\circ}$, and $46.8^{\circ}$, respectively. The central axial channeling effects and the intersecting planes show that a substantial fraction of Er atoms are located on substitutional lattice sites. In order to determine the sublattice preference of Er in AlN, the experimental patterns were fitted to theoretical emission yields, which were calculated by means of the "manybeam" approach, ${ }^{1}$ assuming a perfect crystal lattice. The best fits to the theoretical patterns were obtained for $\mathrm{Er}$ on $\mathrm{Al}$ sites [Figs. 3(e)-3(h)] with the remaining Er atoms occupying random sites. A visual inspection of the patterns already

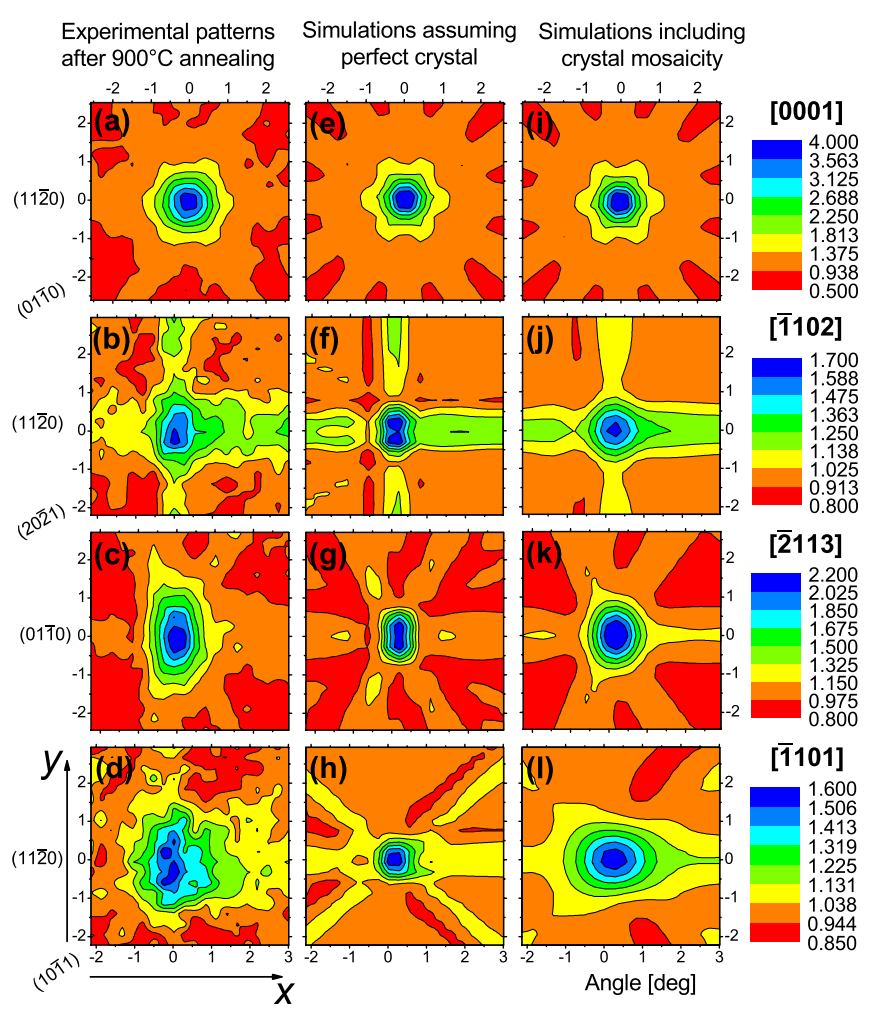

FIG. 3. (a)-(d) Experimental conversion electron emission channeling patterns for ${ }^{167 \mathrm{~m}} \mathrm{Er}$ in AlN following $T_{\mathrm{A}}=900^{\circ} \mathrm{C}$. (e)-(h) Best fits of theoretical emission yields assuming a perfect AlN crystal structure corresponding to, respectively, $61 \%, 28 \%, 29 \%$, and $17 \%$ of Er on Al sites. (i)-(1) Best fits of theoretical patterns including the broadening due to AlN mosaicity, leading to $\mathrm{Er}_{\mathrm{Al}}$ fractions of $69 \%, 60 \%, 64 \%$, and $63 \%$.

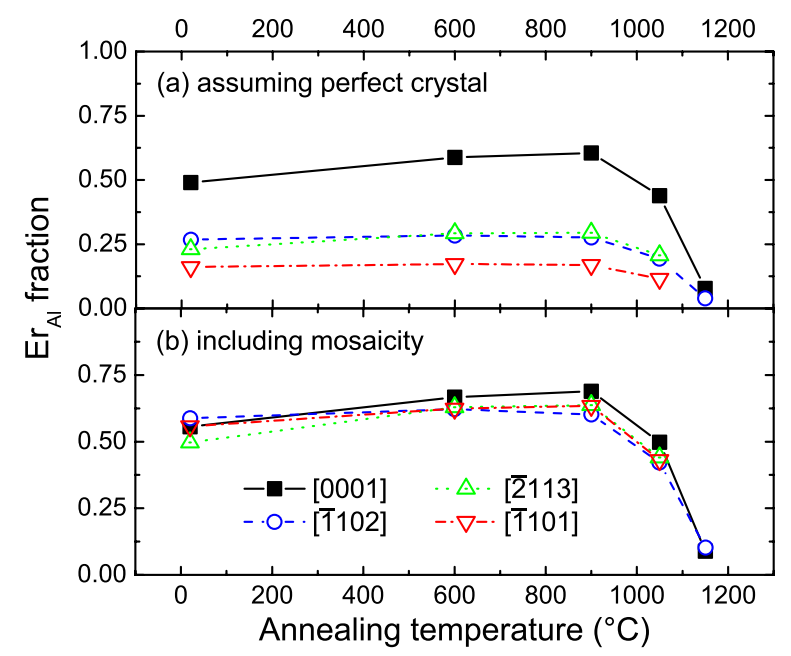

FIG. 4. Fractions of $\mathrm{Er}_{\mathrm{Al}}$ deduced from a fit to (a) theoretical emission yields assuming a perfect crystal and (b) theoretical simulations including the broadening due to mosaicity.

shows that the agreement is rather poor, especially for the axes with a large inclination: The experimental [1101] pattern [Fig. 3(d)] is by far broader than the simulated "best fit" for a perfect crystal [Fig. 3(h)]. Figure 4(a) shows the $\mathrm{Er}_{\mathrm{Al}}$ fractions deduced from this fitting procedure as a function of annealing temperature. A comparison of the results of the four measured axes indicates that the fractions apparently decrease with increasing inclination angle $\theta$. This unphysical effect, however, is an artefact of the progressively worse agreement between theory and experiment.

In order to investigate if this behavior is due to mosaicity, the fitting procedure was repeated with another set of theoretical emission yields that include the mosaic broadening. This was achieved taking the convolution of the simulations for a perfect crystal with the appropriate distribution functions, according to Fig. 1. The widths of the distributions were taken from the values of the fitted curve in Fig. 2 for the corresponding inclination angles. The best fits of these corrected simulations to the experimental patterns are shown in Figs. 3(i)-3(1). It is obvious that these simulations display a significantly better agreement to the experiments than the ones that assume a perfect crystal. Furthermore, as can be seen in Fig. 4(b), the experimental substitutional fractions along the four crystal axes are now equal within the experimental error bars of about $10 \%$. This demonstrates that the broadening of the experimental emission channeling effects can be explained quantitatively by the mosaicity of the layer, as determined with XRD.

Note that in a similar experiment with ${ }^{167 \mathrm{~m}} \mathrm{Er}$ implanted into a GaN film, ${ }^{13,14}$ an almost perfect agreement was found between the experiment and the theoretical simulations assuming a perfect crystal. However, for that particular sample, the tilt $\left(0.08^{\circ}\right)$ and twist $\left(0.19^{\circ}\right)$ values were smaller than the angular resolution $\left(0.24^{\circ}\right)$ of the emission channeling technique. Consequently, the measured mosaic broadening was too small to play a major role, which explains the good correspondence between theory and experiment for GaN. Moreover, our approach allows the extraction of physical information on the lattice site location of Er in the highly mosaic AIN layer. The data in Fig. 4(b) show that already after room-temperature 
implantation, the majority $(\sim 55 \%-60 \%)$ of the Er atoms are located on $\mathrm{Al}$ sites $\left(\mathrm{S}_{\mathrm{Al}}\right)$, with the remainder situated on random sites. Including a third fraction in the fitting procedure does not considerably improve the fit quality, although it cannot be excluded that less than 5\% of the Er atoms are located on other high-symmetry sites. The largest $\mathrm{Er}_{\mathrm{Al}}$ fraction of about $65 \%$ is obtained after vacuum annealing up to $900^{\circ} \mathrm{C}$. However, this fraction decreases after annealing at $1050^{\circ} \mathrm{C}$ in $\mathrm{N}_{2}$ and almost disappears after an additional annealing step at $1150{ }^{\circ} \mathrm{C}$ in $\mathrm{N}_{2}$, which is unexpected, considering that AlN is very resistant to high temperatures. ${ }^{18}$ Ronning et al. ${ }^{19}$ and Lorenz and Vianden ${ }^{20}$ have observed a similar decrease in substitutional fraction for this temperature range in perturbed angular correlation (PAC) experiments on In-implanted AlN, which was attributed to the oxidation of the surface layer.

With the emission channeling technique, one can in principle also deduce the root mean square $(\mathrm{rms})$ displacements of Er atoms from the ideal Al lattice sites. However, the large mosaic broadening leads to a loss of information in the experimental emission patterns of the AIN samples. As a result, it was not possible to deduce accurate rms displacements from this channeling experiment.

Our observations on the lattice site location of ${ }^{167 \mathrm{~m}} \mathrm{Er}$ can be compared with the results of previous emission channeling experiments on rare earths in AlN reported by Vetter et al., ${ }^{21,22}$ where $69 \%-78 \%$ of ${ }^{169 *} \mathrm{Tm}$ and $\sim 57 \%$ of ${ }^{147} \mathrm{Nd}$ were found on $\mathrm{S}_{\mathrm{Al}}$ sites. The AlN layer used in the ${ }^{169 *} \mathrm{Tm}$ experiment was grown on a $6 \mathrm{H}-\mathrm{SiC}$ substrate while the one for ${ }^{147} \mathrm{Nd}$ was deposited on sapphire, as in our case. Since AlN has a much smaller lattice mismatch with $6 \mathrm{H}-\mathrm{SiC}$ than with sapphire, the crystal quality (including mosaicity) of layers grown on $\mathrm{SiC}$ is better than those grown on sapphire substrates. This assumption is supported by the fact that the angular emission spectra from ${ }^{169 *} \mathrm{Tm}$ shown in Ref. 21 do not seem to be broadened by mosaic spread. On the other hand, for their analysis of the ${ }^{147} \mathrm{Nd}$ experiment Vetter et al. noticed a broadening effect due to the sample mosaicity and took this into account by convoluting the patterns with a "resolution function," however, without working out the procedure in detail. ${ }^{22}$ Summarizing both our results and those of Refs. 21 and 22, one may conclude that, although radioactive probes from different elements were used, approximately $60 \%-80 \%$ of implanted rare earths are found to occupy the $\mathrm{Al}$ sites in AlN.

Similar to the emission channeling data presented above, the crystal mosaicity can be taken into account in the $\mathrm{RBS} / \mathrm{C}$ data by a convolution of the theoretical half angle for a perfect crystal $\psi_{1 / 2}^{\mathrm{B}}$ with the angular mosaic spread at the inclination angle $\theta$ of the channeling axis (as determined above). The corrected critical angles $\psi_{1 / 2}^{\text {mos }}$ are listed in the last column of Table I. These values increase with increasing inclination angle $\theta$, like the experimental data, indicating that crystal mosaicity is indeed the driving force behind the broadening of the experimental RBS/C effects.

In conclusion, we have shown that the mosaicity of a hetero-epitaxial film can lead to a broadening and a degradation of experimental emission channeling and RBS/C effects.
We were able to quantitatively model this phenomenon using the results of X-ray rocking curve scans. This approach allowed to accurately determine the lattice site of $\mathrm{Er}$ in AlN, despite the large influence of mosaicity on the channeling measurements. This model is, however, not limited to this specific example: in general, it predicts that any channeling measurements on a sample with a sufficiently large mosaicity will be influenced by this effect, regardless of the specific sample composition or channeling technique that is used. This can lead to erroneous determinations of the lattice site location, if this effect is not taken into account.

This work was funded by the FWO, Vlaanderen, the Portuguese Foundation for Science and Technology (CERN/FP/123585/2011), the KU Leuven (GOA/09/006 and GOA/14/007) and the European Commission through the SPIRIT (Support of Public and Industrial Research using Ion beam Technology, Contract 227012) and ENSAR (European Nuclear Science and Applications Research, Contract 262010) projects.

${ }^{1}$ H. Hofsass and G. Lindner, Phys. Rep. 201, 121 (1991).

${ }^{2}$ A. Dygo, J. Kaczanowski, A. Turos, W. Wesch, K. Gartner, and G. Gotz, Nucl. Instrum. Methods Phys. Res. B 64, 721 (1992).

${ }^{3}$ D. Sigurd, R. W. Bower, W. F. Vanderwe, and J. W. Mayer, Thin Solid Films 19, 319 (1973).

${ }^{4}$ H. Ishiwara, K. Hikosaka, and S. Furukawa, J. Appl. Phys. 50, 5302 (1979).

${ }^{5}$ P. K. Bhattacharya, J. Appl. Phys. 52, 2804 (1981).

${ }^{6}$ R. Samlenski, G. Flemig, R. Brenn, C. Wild, W. Mullersebert, and P. Koidl, J. Appl. Phys. 74, 2134 (1993).

${ }^{7}$ K. Takahiro, S. Nagata, and S. Yamaguchi, Appl. Phys. Lett. 69, 2828 (1996).

${ }^{8}$ R. Moons, S. Blasser, J. Dekoster, A. Vantomme, J. De Wachter, and G. Langouche, Thin Solid Films 324, 129 (1998).

${ }^{9}$ E. V. Monakhov, M. F. Fyhn, and A. N. Larsen, Nucl. Instrum. Methods Phys. Res. B 164-165, 103 (2000).

${ }^{10}$ V. Srikant, J. S. Speck, and D. R. Clarke, J. Appl. Phys. 82, 4286 (1997).

${ }^{11}$ U. Wahl, J. G. Correia, A. Czermak, S. G. Jahn, P. Jalocha, J. G. Marques, A. Rudge, F. Schopper, J. C. Soares, A. Vantomme, P. Weilhammer, and I. Collaboration, Nucl. Instrum. Methods Phys. Res. A 524, 245 (2004).

${ }^{12}$ U. Wahl, A. Vantomme, J. De Wachter, R. Moons, G. Langouche, J. G. Marques, and J. G. Correia, Phys. Rev. Lett. 79, 2069 (1997).

${ }^{13}$ B. De Vries, V. Matias, A. Vantomme, U. Wahl, E. M. C. Rita, E. Alves, A. M. L. Lopes, and J. G. Correia, Appl. Phys. Lett. 84, 4304 (2004).

${ }^{14}$ A. Vantomme, B. De Vries, and U. Wahl, Top. Appl. Phys. 124, 55 (2010).

${ }^{15}$ J. H. Barrett, Phys. Rev. B 3, 1527 (1971).

${ }^{16}$ T. A. Lafford, P. A. Ryan, D. E. Joyce, M. S. Goorsky, and B. K. Tanner, Phys. Status Solidi A 195, 265 (2003).

${ }^{17}$ T. Shibata, K. Asai, S. Sumiya, M. Mouri, M. Tanaka, O. Oda, H. Katsukawa, H. Miyake, and K. Hiramatsu, Phys. Status Solidi C 0, 2023 (2003).

${ }^{18}$ S. Strite and H. Morkoc, J. Vac. Sci. Technol. B 10, 1237 (1992).

${ }^{19}$ C. Ronning, M. Dalmer, M. Uhrmacher, M. Restle, U. Vetter, L. Ziegeler, H. Hofsass, T. Gehrke, K. Jarrendahl, and R. F. Davis, J. Appl. Phys. 87, 2149 (2000).

${ }^{20}$ K. Lorenz and R. Vianden, Phys. Status Solidi C 0, 413 (2002).

${ }^{21}$ U. Vetter, M. F. Reid, H. Hofsass, C. Ronning, J. Zenneck, M. Dietrich, and I. Collaboration, in GaN and Related Alloys, edited by Y. Arakawa, A. Rizzi, J. S. Speck, C. M. Wetzel, and E. T. Yu (Mater. Res. Soc. Symp. Proc., 2003), Vol. 743, p. 417.

${ }^{22}$ U. Vetter, J. B. Gruber, A. S. Nijjar, B. Zandi, G. Oehl, U. Wahl, B. De Vries, H. Hofsaess, M. Dietrich, and I. Collaboration, Phys. Rev. B 74, 205201 (2006). 